\title{
An Improved Three-Phase AMB Distribution System State Estimator
}

DOI:

10.1109/TPWRS.2016.2590499

\section{Document Version}

Accepted author manuscript

Link to publication record in Manchester Research Explorer

\section{Citation for published version (APA):}

Almeida, M. C. D., \& Ochoa, L. (2016). An Improved Three-Phase AMB Distribution System State Estimator. I E E E Transactions on Power Systems. https://doi.org/10.1109/TPWRS.2016.2590499

\section{Published in:}

I E E E Transactions on Power Systems

\section{Citing this paper}

Please note that where the full-text provided on Manchester Research Explorer is the Author Accepted Manuscript or Proof version this may differ from the final Published version. If citing, it is advised that you check and use the publisher's definitive version.

\section{General rights}

Copyright and moral rights for the publications made accessible in the Research Explorer are retained by the authors and/or other copyright owners and it is a condition of accessing publications that users recognise and abide by the legal requirements associated with these rights.

\section{Takedown policy}

If you believe that this document breaches copyright please refer to the University of Manchester's Takedown Procedures [http://man.ac.uk/04Y6Bo] or contact uml.scholarlycommunications@manchester.ac.uk providing relevant details, so we can investigate your claim.

\section{OPEN ACCESS}




\title{
An Improved Three-Phase AMB Distribution System State Estimator
}

\author{
Madson C. de Almeida, Member, IEEE, and Luis F. Ochoa, Senior Member, IEEE
}

\begin{abstract}
State estimators (SEs) are required to enable the evolving and increasingly important role of communications and control in smart distribution systems. In this context, this paper presents an improved three-phase admittance matrix-based (AMB) SE for medium voltage systems to tackle issues related to zero injections, consistency, and the inclusion of voltage measurements. Here, the state variables are the real and imaginary parts of the complex bus voltages, while power and voltage measurements are converted into equivalent currents and voltages, respectively. The key improvements include: (i) considering zero injections through a linear non-weighted procedure, (ii) using phasor rotation for calculation of the equivalent voltage measurements, and, (iii) including the covariance between real and imaginary parts of equivalent current measurements. Despite these new characteristics, the proposed improved AMB SE (ISE) features constant coefficient matrices, thus resulting in reduced computational times. The performance of the ISE is assessed considering a real UK medium voltage system. Its consistency is assessed via a Monte Carlo analysis. Comparisons with other AMB SEs, demonstrate that the proposed three-phase ISE is more robust, statistically more consistent and computationally very competitive.
\end{abstract}

Index Terms-Consistency, distribution systems, equivalent voltage measurements, state estimation, zero injections.

\section{NOMENCLATURE}

$p_{k}^{\text {meas }}$ Active power measurement for any phase of bus $k$;

$q_{k}^{\text {meas }}$ Reactive power measurement for any phase of bus $k$;

$p_{k m}^{\text {meas }}$ Active power measurement for any phase of branch $\mathrm{km}$;

$q_{\mathrm{km}}^{\text {meas }}$ Reactive power measurement for any phase of branch $\mathrm{km}$;

$V^{\eta} \quad$ Vector of three-phase voltages of all buses in iteration $\eta$;

$V_{k}^{\eta} \quad$ Vector of three-phase voltages of bus $k$ in iteration $\eta$;

$v_{k}^{\eta} \angle \theta_{k}^{\eta}$ Voltage in polar form for any phase of bus $k$ in iteration $\eta$;

$v_{k}^{\text {meas }}$ Voltage magnitude measured in any phase of bus $k$;

$Y \quad$ Three-phase admittance matrix of the system;

$Y_{k m}^{s} \quad$ Three-phase series admittance matrix for branch $\mathrm{km}$;

$Y_{k m}^{s h} \quad$ Three-phase shunt admittance matrix for branch $\mathrm{km}$;

$E_{o} \quad$ Vector of three-phase voltages on buses with zero injections;

$E_{n} \quad$ Vector of three-phase voltages on buses with nonzero injections.

This work has been partially funded by FAPESP, CNPq and CAPES

Madson C. de Almeida is with the Department of Systems and Energy, State University of Campinas, Campinas, São Paulo, Brazil (e-mail: madson@dsee.fee.unicamp.br). Luis F. Ochoa is with the School of Electrical and Electronic Engineering, The University of Manchester, Manchester M13 9PL, UK (e-mail: luis_ochoa@ieee.org).

\section{INTRODUCTION}

$\mathrm{T}^{\circ}$ O facilitate the transition towards much more observable and controllable smart distribution systems -part of the Smart Grid vision- and, thus, enabling applications such as power flow management, feeder reconfiguration, optimal voltvar control, fault location, self-healing systems, demand-side management, energy loss reduction, identification of nontechnical losses, it is critical to determine the most likely state of the system during operation [1], [2]. The latter is achieved using state estimators (SEs) [3], [4], a fundamental part of modern Distribution Management System (DMS) [1], [2], [5].

In transmission systems, typically with higher levels of monitoring and balanced loads, SEs can be regarded as consolidated tools. However, the required maturity level has not yet been reached in distribution systems given that some of their features pose significant challenges to SEs. Indeed, distribution systems have limited monitoring, making it necessary to rely on the so-called pseudo-measurements. Moreover, due to typical high $\mathrm{r} / \mathrm{x}$ ratios, load unbalances, nontransposed lines and the presence of single, two and threephase laterals, distribution systems generally require a threephase modelling with all phase quantities as well as phase couplings represented [6], [7]. As a result, the number of state variables and measurements involved in the state estimation process increase, making it more difficult to solve and more time consuming.

The most traditional state estimation approach considers power and voltage magnitudes as measurements or pseudomeasurements, and complex bus voltages in polar coordinates as state variables [3], [4]. This approach requires the coefficient matrices to be processed iteratively, thus increasing computational time. The simplest and most common formulation of the traditional SE is based on the normal equation (NE). However, due to the need for representing injections in buses without loads and generators, known as zero injections, convergence problems can occur in the NE formulation. To prevent this and improve accuracy, the normal equation with constraints (NE/C) or the Hachtel Tableau are approaches adopted when equality constraints need to be represented [3], [4], [9], [10], [10].

Although future distribution systems are likely to have much higher levels of monitoring than today, pseudomeasurements and zero injections will still be required for state estimation [1], [2]. Pseudo-measurements are traditionally obtained from historical data or customer demand surveys, resulting in poor accuracy [11]. Although their accuracy is expected to increase by aggregating information from smart meters [1], they are likely to still be an issue. In 
addition, adequate modelling of zero injections is also crucial: they are perfect information and do not require monitoring, so they can significantly improve the overall accuracy of the estimates [8], [9]. Modelling both pseudo-measurements and zero injections is, nonetheless, a challenge for distribution SEs, particularly when using the NE.

In the NE formulation, the poorer the accuracy of pseudomeasurements, the smaller the weights associated to them. Conversely, large weights are associated to the perfect zero injections. Due to the highly contrasting weights, this can result in ill-conditioned coefficient matrices and deteriorated convergence [3], [4], [8], [9]. To tackle this, the NE/C or the Hachtel Tableau are commonly used. In these formulations the zero injections are not weighted, therefore, the ill-conditioning problems are not prone to occur. Nonetheless, despite the benefits, they result in indefinite coefficient matrices, which require special factorization techniques and, hence, increase the complexity of implementing the SEs [10], [12].

Based on the above, state estimation approaches that result in constant and better conditioned coefficient matrices become more appealing for distribution systems. In this context, the Admittance Matrix-Based (AMB) SE using the NE was proposed in [13], resulting in a constant coefficient matrix. To reduce the computational time, a fast decoupled AMB SE was proposed in [14], pairing measurements and simplifying the constant coefficient matrix. To further improve the accuracy and the convergence of the AMB, in [9] zero injections where catered for by adopting the NE/C formulation and, therefore, special factorization techniques were required.

This paper presents an improved AMB state estimator (ISE) for medium voltage distribution systems, thus the state variables are the real and imaginary parts of the complex bus voltages, while the power and voltage measurements are converted into the equivalent currents and voltages, respectively. In particular, the key contributions include: $(i)$ the zero injections are considered through a linear nonweighted procedure, resulting in a constant positive definite coefficient matrix, which requires only classical factorization techniques and prevent convergence problems; (ii) the concept of phasor rotation is used to obtain the three-phase equivalent voltage measurements, improving the convergence characteristics of the ISE; and, (iii) the covariance between the real and imaginary parts of the equivalent current measurements are considered, improving the statistical consistence of the ISE. A three-phase modelling in phase coordinate is adopted to consider all phase quantities as well as their couplings [6], [7]. As a consequence, unsymmetrical systems as well as unbalanced loads can be handled.

The Monte Carlo analysis is used to evaluate the robustness and the consistency of the ISE. Comparisons with the AMB $\mathrm{NE}$ and the AMB NE/C SEs highlight the benefits of the ISE. The performance of the ISE is assessed considering a threephase real UK medium voltage system.

In the next section, the ISE is presented and the proposed improvements are described in detail. Section IV introduces the concept of statistical consistency and shows the procedure to assess a SE. The results from the case studies are presented and discussed in section V. Finally, in section VI the main conclusions are drawn.

\section{IMPROVED AMB STATE ESTIMATOR (ISE)}

In state estimation, the topology of the system is typically assumed known and correct [3]. Therefore, given a set containing actual measurements, pseudo-measurements, and zero injections, as well as the corresponding variances, an SE provides the most likely estimate for the state variables [3], [4]. In cases where the system topology needs to be estimated, breakers/switches can be modelled [3], [15], [16]; however, this is outside the scope of this paper.

In the traditional SE, the active and reactive powers as well as voltage magnitudes are the measurements, while complex bus voltages in polar coordinates are the state variables. The measured values $z$ relate the state variables $x$ through the measurement functions $h(x)$ and the vector of measurement errors, as in (1).

$$
z=h(x)+e
$$

The estimate for $x$ can be obtained via the Weighted Least Squares (WLS) approach minimizing the index $J(x)$, where $W$ is the diagonal weight matrix [3], [4].

$$
\min J(x)=\frac{1}{2}[z-h(x)]^{T} W[z-h(x)]
$$

Applying the Gauss-Newton method to (2), the estimate for $x$ is obtained solving iteratively the normal equation (3), where $H(x)$ is the Jacobian matrix of $h(x)$ and $H(x)^{T} W H(x)$ is the Gain matrix.

$$
\begin{aligned}
H(x)^{T} W H(x) \Delta x & =H(x)^{T} W[z-h(x)] \\
x^{\eta+1} & =x^{\eta}+\Delta x
\end{aligned}
$$

In a conventional AMB SE, the measurements $z$ are converted into real and imaginary parts of equivalent currents and equivalent voltages, $Z$. The state variables $x$ are the complex bus voltages in rectangular coordinates. Thus, a WLS solution can be obtained via the Gauss-Newton method solving iteratively the normal equation (4). In (4), unlike (3), the gain matrix $H^{T} W H$ is constant, therefore, it is built and factorized just once during the iterative solution.

$$
\begin{gathered}
H^{T} W H \Delta x=H^{T} W[Z-h(x)] \\
x^{\eta+1}=x^{\eta}+\Delta x
\end{gathered}
$$

In the proposed ISE, the state variables are the real and imaginary parts of the complex bus voltages. The available active and reactive powers as well as voltage magnitudes are converted into real and imaginary parts of equivalent currents and real parts of equivalent voltages, respectively. Therefore, the set of equivalent measurements, $Z$, relate the real and imaginary parts of the bus voltages, $V$, via a constant Jacobian matrix, $H$, as in (5).

$$
Z=H V
$$

In this section, the mathematical formulation of the proposed ISE is described. This includes the adopted approach to deal with zero injections as well as the process to obtain the equivalent current and voltage measurements, and the corresponding variances, covariances and weights. The threephase representation in phase coordinate adopts the classical 
unbalanced three-phase models and the corresponding power flow equations from [6] and [7]. Despite the changes in the variables required for the $\mathrm{AMB}$, all the system devices are modelled as in traditional unbalanced three-phase SEs.

\section{A. Considering Zero Injections Using a Linear Approach}

In order to deal with zero injections, here it is proposed to eliminate the variables associated to them. This concept is similar to that proposed in [11] where buses associated to zero injections were eliminated. However, in that case, given that the SE could only provide complex voltages for the remaining buses, one power flow run was needed to calculate the state variables of the eliminated buses. The approach proposed in this paper allows the ISE to estimate all the state variables and therefore no additional power flow is required. Further advantages of the proposed approach on the resulting coefficient matrices include:

- Positive definite. This means specialized factorization techniques are not required.

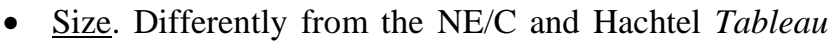
formulations [3], [4], [9], the size of the coefficient matrices does not increase.

- Sparsity. Since distributions systems are typically radial or weakly meshed, the resulting coefficient matrices remain highly sparse.

To eliminate the state variables associated with the zero injections (5) has to be partitioned as in (6), where $Z_{o}$ contains the real and imaginary parts of the zero injections and $E_{o}$ contains the real and imaginary parts of the voltages on buses containing zero injections. All these vectors and matrices are three-phase in phase coordinate.

$$
Z=\left[\begin{array}{l}
Z_{o} \\
Z_{n}
\end{array}\right]=\left[\begin{array}{l}
H_{o} \\
H_{n}
\end{array}\right]\left[\begin{array}{l}
E_{o} \\
E_{n}
\end{array}\right]=\left[\begin{array}{ll}
H_{o o} & H_{o n} \\
H_{n o} & H_{n n}
\end{array}\right]\left[\begin{array}{c}
E_{o} \\
E_{n}
\end{array}\right]
$$

Solving (6) for $Z_{o}$ (equal to zero) allows obtaining the voltages for the buses with zero injections as shown in (7). Note that this equation is linear given that the equivalent measurements and the state variables are in rectangular form [9]. Furthermore, in (7), the number of variables to be calculated is equal to the number of zero injections, i.e., the number of unknowns is equal to the number of known quantities. Additionally, the coefficient matrix $H_{o o}$ is full rank. Thus, null injections are guaranteed to be catered for [3], [4].

$$
E_{o}=-H_{o o}^{-1}\left[H_{o n} E_{n}\right]
$$

Considering (7) and (6), $Z_{n}$ can be obtained as (8). Note that the resulting coefficient matrix $A$ is constant and the equivalent measurements $Z_{n}$ depend on the complex voltages $E_{n}$.

$$
Z_{n}=\left[-H_{n o} H_{o o}^{-1} H_{o n}+H_{n n}\right] E_{n}=A E_{n}
$$

Given (7) and (8), the voltages $E_{n}$ and $E_{o}$ can be obtained via the WLS optimization problem (9).

$$
\begin{gathered}
\min J(x)=\frac{1}{2}\left[Z_{n}-A E_{n}\right]^{T} W\left[Z_{n}-A E_{n}\right] \\
\text { s.t. } E_{o}=\left[-H_{o o}^{-1} H_{o n}\right] E_{n}
\end{gathered}
$$

Applying the Gauss-Newton method to (9), the voltages $E_{n}$ and $E_{o}$ are obtained solving iteratively the normal equation (10), where $W$ is the constant weight matrix of the equivalent measurements in $Z_{n}, A^{T} W A$ is a constant Gain matrix, and $\eta$ indicates the current iteration.

$$
\begin{gathered}
\Delta E_{n}^{\eta}=\left[A^{T} W A\right]^{-1} A^{T} W\left[Z_{n}^{\text {meas }}\left(V^{\eta}\right)-Z_{n}^{\text {calc }}\left(V^{\eta}\right)\right] \\
\Delta E_{n}^{\eta+1}=E_{n}^{\eta}+\Delta E_{n}^{\eta}
\end{gathered}
$$

Finally, replacing $Z_{n}^{\text {calc }}$ in (10) by (8) allows obtaining the voltages for the next iteration, $\eta+1$, in a more direct fashion instead of using (10) as demonstrated below.

$$
\begin{gathered}
\Delta E_{n}^{\eta}=\left[A^{T} W A\right]^{-1} A^{T} W\left[Z_{n}^{\text {meas }}\left(V^{\eta}\right)-Z_{n}^{\text {calc }}\left(V^{\eta}\right)\right] \\
\Delta E_{n}^{\eta}=\left[A^{T} W A\right]^{-1} A^{T} W\left[Z_{n}^{\text {meas }}\left(V^{\eta}\right)-A E_{n}^{\eta}\right] \\
\Delta E_{n}^{\eta}=\left[A^{T} W A\right]^{-1} A^{T} W Z_{n}^{\text {meas }}\left(V^{\eta}\right)-E_{n}^{\eta} \\
\Delta E_{n}^{\eta}+E_{n}^{\eta}=\left[A^{T} W A\right]^{-1} A^{T} W Z_{n}^{\text {meas }}\left(V^{\eta}\right) \\
E_{n}^{\eta+1}=\left[A^{T} W A\right]^{-1} A^{T} W Z_{n}^{\text {meas }}\left(V^{\eta}\right)
\end{gathered}
$$

Consequently, the proposed formulation to estimate the state variables involves only (7) and (11), which corresponds to a more computationally efficient process (and simpler to implement) given that $Z_{n}^{\text {calc }}$ is not calculated at each iteration. In addition, given that in (7) there are no weights associated to zero injections, ill-conditioning problems can be prevented.

\section{B. Solution Algorithm}

In (7) and (11) the coefficient matrices $A^{T} W A$ and $H_{o o}$ are constant and, therefore, they are built and factorized just once. Moreover, these matrices are highly sparse, since distribution systems are typically radial or weakly meshed. As a consequence, the proposed ISE can be implemented in a very efficient way using classical and well-established techniques for sparse storage, pivoting and factorization.

The solution algorithm for the proposed ISE is illustrated in Fig. 1. First, an initial guess for voltages $E_{n}^{0}$ is specified (e.g., three-phase flat start [6] or the last estimated state). Then, the matrices $H_{o o}$ and $A^{T} W A$ are built and factorized. $E_{o}^{0}$ is obtained with (7). Next, with the most recently calculated voltages within the iterative process, $E_{n}^{\eta+1}$ and $E_{o}^{\eta+1}$ are calculated using (11) and (7), respectively. This step is repeated until the maximum absolute increment in voltages on buses without zero injections is less than or equal to a tolerance $\tau$.

In this paper, the initial guess corresponds to $1.0 \mathrm{pu}$ for voltage magnitudes shifted by $120^{\circ}$ per phase (flat start). The adopted tolerance is equal to $10^{-5} \mathrm{pu}$.

\section{Equivalent Current Measurements}

In order to convert power measurements into equivalent current measurements it is assumed that both active and reactive power measurements are available. Thus, the real and imaginary parts of the corresponding current can be obtained using (12) and (13). Given that the latter requires the most 


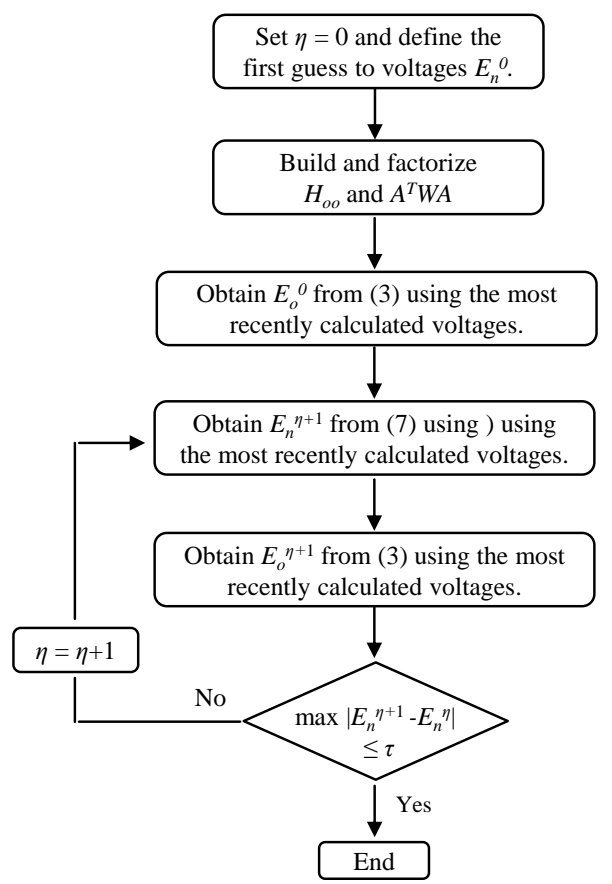

Fig. 1. Solution algorithm for the proposed ISE.

$$
\begin{aligned}
\vec{l}_{k m}^{e q} & =i_{k m}^{\text {real }}+j i_{k m}^{\text {imag }}=\left[\frac{p_{k m}^{\text {meas }}+j q_{k m}^{\text {meas }}}{v_{k}^{\eta} \angle \theta_{k}^{\eta}}\right] \\
\vec{l}_{k}^{e q} & =i_{k}^{\text {real }}+j i_{k}^{\text {imag }}=\left[\frac{p_{k}^{\text {meas }}+j q_{k}^{\text {meas }}}{v_{k}^{\eta} \angle \theta_{k}^{\eta}}\right] \\
\vec{v}_{k}^{e q} & =v_{k}^{\text {real }}+j v_{k}^{\text {imag }}=v_{k}^{\text {meas }}\left[\frac{v_{k}^{\eta} \angle \theta_{k}^{\eta}}{v_{k}^{\eta}}\right] \\
v_{k}^{e q}= & R e\left\{\vec{v}_{k}^{e q} e^{-j \phi_{k}}\right\}=v_{k}^{\text {real }} \cos \phi_{k}+v_{k}^{\text {imag }} \sin \phi_{k}
\end{aligned}
$$

recently calculated voltages, these equivalent measurements need to be updated at each iteration. Note that by considering the real and imaginary parts of the calculated currents as equivalent measurements, the observability of the system and the redundancy of the measurements remain the same.

It is worth noting that according to (12) and (13) a pair of active and reactive power measurements (for a given phase) are required to obtain the equivalent current measurements. However, if any of two power measurements is missing, a pseudo-measurement with adequate variance can be adopted.

\section{Equivalent Voltage Measurements via Phasor Rotation}

Equation (14) is usually adopted to convert voltages magnitude measurements into equivalent complex voltage measurements [9], [13], [14]. However, given that a magnitude measurement is converted into a pair of equivalent measurements (real and imaginary parts of the equivalent complex voltage), the observability of the system and the redundancy of the measurements set are artificially improved. This, in turn, can have negative effects on the convergence of the AMB SE, as demonstrated in [17] for a single-phase distribution power system. In this case, a simple but effective solution is to discard the imaginary part of the equivalent complex voltages. Nonetheless, this solution cannot be directly applied to three-phase estimators.

In the context of three-phase SEs, here it is proposed to first obtain the complex voltages $\vec{v}_{k}^{e q}$ for each phase of bus $k$ according to (14) and then rotate these voltages by an angle $\phi_{k}$ that brings them close to the real positive axis of the complex plane, thus obtaining $\vec{v}_{k}^{e q} e^{-j \phi_{k}}$. The equivalent voltage measurements proposed in this paper are the real part of these rotated complex voltages, as in (15). Fig. 2 illustrates this procedure for a voltage magnitude measured in phase $c$ of bus $k$. The dashed circle indicates the locus of the voltage magnitude measurement $v_{k}^{\text {meas }}$. The value for the angle $\phi_{k}$ corresponds to that adopted for $\theta_{k}$ at the flat start (which caters for any angular displacement resulting from three-phase transformer connections). Therefore, $\phi_{k}$ is constant and, if no angular displacement exists, the corresponding angles for phases $a, b$ and $c$, will be $0^{\circ},-120^{\circ}$ and $120^{\circ}$, respectively. Thereby, during the state estimation process the magnitudes of the proposed equivalent voltages (15) will be close to the measured voltages $v_{k}^{\text {meas }}$, whereas the imaginary parts of the rotated complex voltages, $\operatorname{Im}\left\{\vec{v}_{k}^{e q} e^{-j \phi_{k}}\right\}$, will be close to zero. Since only the real part of the rotated voltage is used, the observability of the system and the redundancy of the measurements set are maintained while, crucially, the convergence features of the ISE are significantly improved.

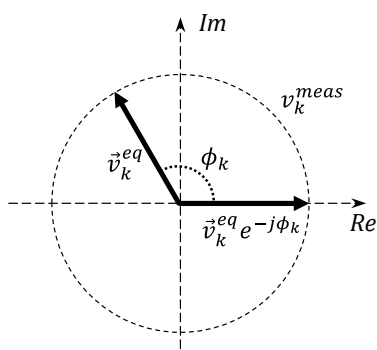

Fig. 2. Equivalent voltage measurement in the complex plane (phase $c$ ).

\section{E. Variances of Measurements}

The variances of equivalent measurements are calculated from variances of the measurements as well as considering error propagation theory [5]. Typically, variances of the measurements are assumed to be fixed values according to the type of the measurement or proportional to the measured values [18], [18]. For the sake of simplicity, in this paper the variance of the $i$-th measurement or pseudo-measurement will be proportional to the measured values according to (16), where $z_{i}$ is the value for the $i$-th measurement or pseudomeasurement and $\varepsilon$ is the percentage error attributed to each type of measurement or pseudo-measurement.

$$
\sigma_{i}^{2}=\left[\frac{z_{i} \times \varepsilon}{3 \times 100}\right]^{2}
$$

\section{F. Variances for Equivalent Voltage Measurements}

To allocate adequate variances to the equivalent voltage measurements prior to the SE, knowledge of complex voltages is required. Then, considering the error propagation theory and given the variance of a voltage measurement, the variance for the equivalent voltage measurement $v_{k}^{e q}$, for any phase, is 
calculated by (17), where $e_{k}+j f_{k}$ is the complex voltage (for a given phase) regarding the bus $k$ calculated from a power flow [13]. The complex voltages $e_{k}+j f_{k}$ can also be obtained from the previous estimation.

$$
\begin{aligned}
\sigma_{v_{k}^{e q}}^{2} & =\left[\frac{e_{k}}{\sqrt{e_{k}^{2}+f_{k}^{2}}} \cos \phi_{k}\right. \\
+ & \left.\frac{f_{k}}{\sqrt{e_{k}^{2}+f_{k}^{2}}} \sin \phi_{k}\right]^{2} \sigma_{v_{k}^{\text {meas }}}^{2}
\end{aligned}
$$

\section{G. Variances and Covariances for Equivalent Current Measurements}

In the conventional AMB SE, for the sake of simplicity, usually the correlations between the real and imaginary parts of the equivalent current measurements are not considered, i.e., the corresponding covariances are neglected [20]. However, as it will be demonstrated in section V.D, this simplification can significantly deteriorate the consistency of the AMB SE. Therefore, here it is proposed to consider the covariances between real and imaginary parts of the equivalent current measurements in order to improve the consistency of the ISE.

Given the measurements variances and considering the error propagation theory, the variances/covariances of the real and imaginary parts of the equivalent currents for a given phase of the distribution system are calculated by (18). The same equation is applied to the current flows by simply considering power flows instead of power injections.

$$
\begin{aligned}
& {\left[\begin{array}{cc}
\sigma_{i_{k}^{\text {real }}}^{2} & \sigma_{i_{k}^{\text {real }}}^{\text {imag }} \\
\sigma_{i_{k}^{\text {real }} i_{k}^{\text {imag }}} & \sigma_{i_{k}^{\text {imag }}}^{2}
\end{array}\right]=} \\
& {\left[\begin{array}{cc}
c^{2} \sigma_{p_{k}^{\text {meas }}}^{2}+d^{2} \sigma_{q_{k}^{\text {meas }}}^{2} & c d\left[\sigma_{p_{k}^{\text {meas }}}^{2}-\sigma_{q_{k}^{\text {meas }}}^{2}\right] \\
c d\left[\sigma_{p_{k}^{\text {meas }}}^{2}-\sigma_{q_{k}^{\text {meas }}}^{2}\right] & d^{2} \sigma_{p_{k}^{\text {meas }}}^{2}+c^{2} \sigma_{q_{k}^{\text {meas }}}^{2}
\end{array}\right]}
\end{aligned}
$$

The constants $c$ and $d$ in (18) are defined as bellow and are calculated only once for each state estimation run.

$$
c=\frac{e_{k}}{e_{k}^{2}+f_{k}^{2}} \text { and } d=\frac{f_{k}}{e_{k}^{2}+f_{k}^{2}}
$$

\section{H. Weights for Equivalent Measurements}

In the ISE, the weighting matrix $W$ is a square matrix whose rows and columns are associated with equivalent measurements. The diagonal element $W_{i, i}$ contains the weight associated to the $i$-th equivalent measurement while the offdiagonal element $W_{i, j}$ contains the weight relating the $i$-th and the $j$-th equivalent measurements. The weights for the equivalent voltage measurements are the inverse of the variances (17). Given that the real and imaginary parts of the equivalent current measurements are correlated for a given phase, i.e., the corresponding covariances cannot be neglected, the inverse of the covariance matrix (18) produces two diagonal and two off-diagonal elements in the weighting matrix. $W$ is constant and, therefore, needs to be calculated only once for each SE run.

\section{CONSISTENCY OF StATE Estimators}

SEs must be consistent in that the estimated values and variances have to be in agreement with the measurements and their specified variances. Thereby, in a consistent SE, the smaller the estimated variances the closer are the estimates from their expected values.

The concept of consistency was first applied in [18] to statistically assess a power system SE. It was concluded that the single-phase traditional WLS NE state estimator is consistent and suitable for estimating the state of balanced medium voltage power systems. For the single-phase WLS AMB NE state estimator, however, it was shown in [17] that some improvements are required to make it consistent.

In order to carry out an adequate consistency assessment for the proposed ISE, a Monte Carlo analysis is required. For a consistent ISE, the mean value of the consistency index (19) will tend to the number of state variables minus the number of zero injections. In (19), the hat stands for the estimated state, while the bar stands for the true state obtained from a power flow run. In addition, for a specified confidence level $\alpha$, for instance $95 \%$, the ISE must have a consistency index between the bounds shown in (20) [21].

$$
\begin{gathered}
\mu=\left[\bar{E}_{n}-\hat{E}_{n}\right] H_{n}^{T} W H_{n}\left[\bar{E}_{n}-\hat{E}_{n}\right] \\
\chi_{n}^{2}\left[\frac{1-\alpha}{2}\right] \text { and } \chi_{n}^{2}\left[\frac{1+\alpha}{2}\right]
\end{gathered}
$$

Fig. 3 illustrates the adopted procedure to statistically assess consistency. First, error-free values obtained from a power flow are used as true measurements, $z^{\text {true }}$. Then, random errors following a normal distribution are added to these true values according to (21), where $b$ is a normally distributed random variable with zero mean and unitary variance and $\sigma$ is a vector containing the standard deviation of the measurements and pseudo-measurements. The resulting values correspond to measurements, $z^{\text {meas }}$, that are then used to calculate equivalent measurements. Finally, the estimated state is obtained with the proposed ISE. The consistency index (19) is computed from the ISE estimated values and the bounds (20) are obtained from the chi-square distribution for a given confidence level.

$$
z^{\text {meas }}=z^{\text {true }}+b \sigma
$$

\section{CASE Studies}

This section presents case studies considering a real threephase urban medium voltage distribution system with unbalanced loads from the North West of England. First, the convergence and the quality of the estimates are evaluated considering error-free values for measurements and pseudomeasurements. Then, the influence of the variances calculated for the equivalent measurements are assessed. Finally, the consistency and some fundamental computational features of the ISE are evaluated. To show the corresponding benefits, the ISE is compared to two well-known formulations of the AMB SE: the AMB NE SE and the AMB NE/C SE. Three-phase voltages are shown as well. 


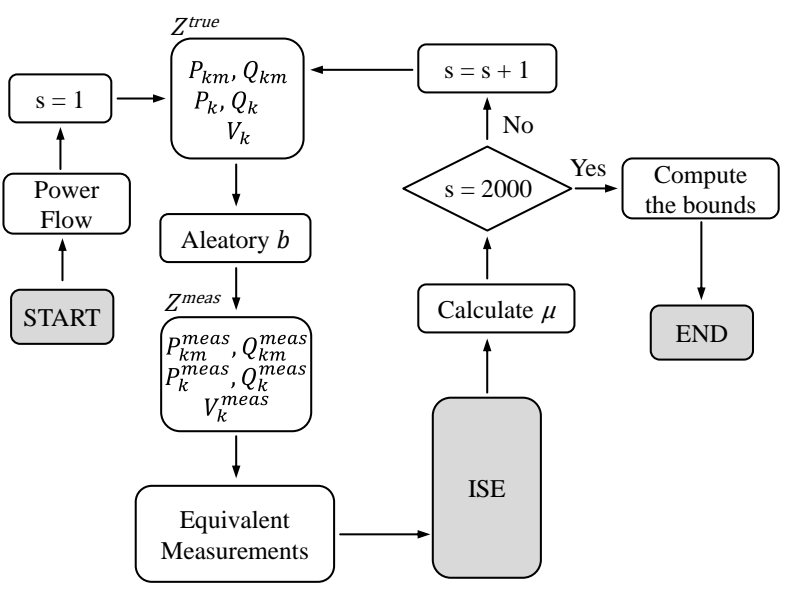

Fig. 3. Procedure for consistency assessment.

\section{A. Real Medium Voltage Distribution System}

The real $11 \mathrm{kV}$ radial urban distribution system shown in Fig. 4 as well as the corresponding monitoring considerations are presented in this section. The system contains 10 feeders supplying approximately 12,000 customers by $8911 / 0.433 \mathrm{kV}$ secondary transformers and 48 nodes with zero injection, i.e., a total of $89 \times 6+48 \times 6=822$ state variables (considering real and imaginary parts per phase). The system is supplied by two 14 MVA $33 / 11 \mathrm{kV} \Delta: \mathrm{Y}$ transformers at the primary substation and has a peak load of 23.28 MW and 5.11 Mvar.

\section{B. Measurement Cases}

Two set of measurements are investigated. In Case I it is assumed there is only a monitor at the busbar of the primary substation. In Case II additional monitors located at the primary side of secondary transformers are considered for the 20 most loaded ones. Therefore, secondary transformers are not modelled. Monitors are able to provide three-phase voltage magnitudes as well as power flows for each of the branches connected to the monitored bus. Power injection pseudomeasurements are considered for all buses for both cases. The errors for voltage measurements, power flow measurements, and power injection pseudo-measurements are assumed to be $0.5,3$, and $30 \%$, respectively. These errors are used to calculate the variances of the measurements according to (16).

\section{Convergence Analysis}

In this section, the convergence of the ISE is compared to the convergence of the conventional AMB NE SE. In the AMB NE SE the real and imaginary parts of the equivalent voltage measurements are calculated using (14). The weights for the equivalent measurements are calculated considering the state obtained from a power flow during peak load. To clearly check convergence, error-free values for measurements and pseudo-measurements are assumed for both estimators. The weights for the zero injections in the conventional AMB NE $\mathrm{SE}$ are set to $10^{6}$. Variances of measurements and pseudomeasurements are obtained according to (16).

Fig. 5 shows the maximum increment in state variables at each iteration for the ISE and AMB NE considering the two sets of measurements (Case I and Case II) and peak load

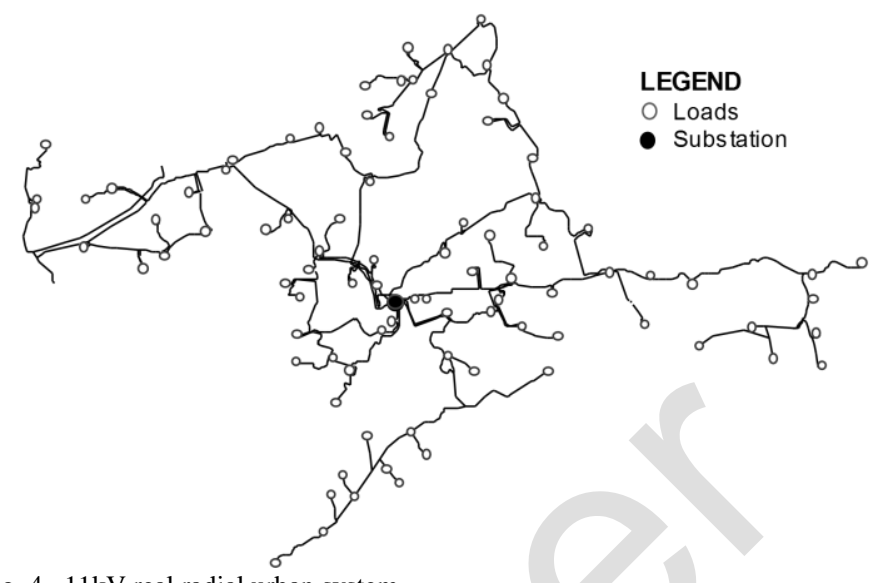

Fig. 4. $11 \mathrm{kV}$ real radial urban system.

condition. For instance, assuming a tolerance $\tau=10^{-3}$, the ISE reaches convergence in 3 iterations whereas the AMB NE requires 9 and 14 iterations, for Case I and Case II, respectively. The slow convergence for the AMB NE is due to the equivalent voltage measurements calculated by (14). In the ISE, the usage of the proposed equivalent voltage measurements (15) solves this issue, making the approach much more robust and efficient. Although not shown here, a similar behavior has been found when fixed variances are assumed for measurements and pseudo-measurements instead of using (16).

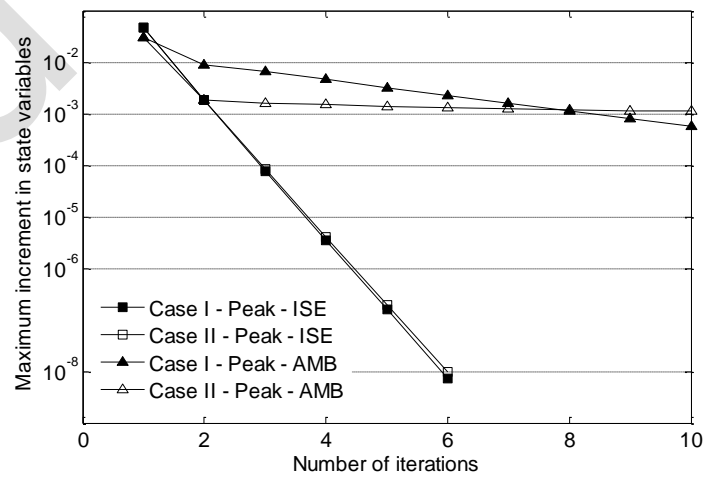

Fig. 5. Maximum increment in state variables per iterations for the ISE and AMB NE. Initialization with flat start and error-free measurements.

For completeness, Fig. 6 shows the three-phase magnitude voltages estimated with the ISE for the second set of measurements (Case II). As expected, the voltages are unbalanced. In general, the more unbalanced are the loads and the more unsymmetrical are the system parameters, the more unbalanced are the voltages.

\section{Quality of the Estimates}

In order to verify the quality of the estimates provided by the ISE, Table I shows the maximum estimated error for complex voltages, the maximum increment in state variables at the convergence point, and the maximum estimated error for zero injections, assuming the previous conditions (including error-free measurements) and $\tau=10^{-5}$. Given that the maximum error in the estimated voltages is small, the estimated state is very close to the actual state (provided by the power flow). Moreover, the zero injections, $Z_{o}$, are satisfied as their maximum estimated error is also very small 


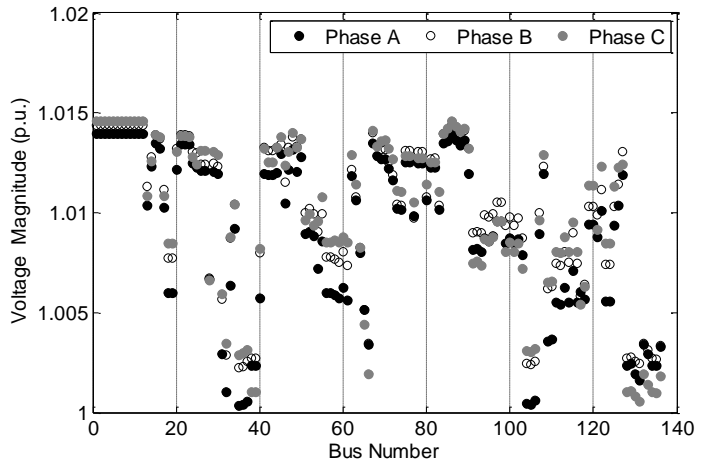

Fig. 6. Three-phase voltage magnitudes estimated with the ISE. Case II with error-free measurements.

TABLE I

Maximum values for some convergence indices for the ISE with $\tau=10^{-5}$

\begin{tabular}{c|c|c|c}
\hline & $\left|\bar{E}_{n}-\hat{E}_{n}\right|$ & $\left|\Delta E_{n}\right|$ & $\left|\bar{Z}_{o}-\hat{Z}_{o}\right|$ \\
\hline Case I & $1.5 \times 10^{-7}$ & $3.4 \times 10^{-6}$ & $4.0 \times 10^{-9}$ \\
\hline Case II & $1.9 \times 10^{-7}$ & $4.0 \times 10^{-6}$ & $1.9 \times 10^{-9}$ \\
\hline
\end{tabular}

$\left(4.0 \times 10^{-9}\right)$. The ISE reached convergence in 4 iterations for both Case I and Case II.

\section{E. Influence of the Variances for Equivalent Measurements}

According to (17) and (18), the variances and covariances and, therefore, the weights of the equivalent measurements depend on the voltages $e_{k}$ and $f_{k}$. Since, in practice, these voltages are likely to be obtained from a previously estimated state with a relatively similar load level (e.g., $15 \mathrm{~min}$ before), it is crucial to investigate the impact of these voltages.

Fig. 7 shows the maximum increment in the state variables per iteration considering Case I and Case II as well as two different load levels to obtain voltages $e_{k}$ and $f_{k}$ : peak and $90 \%$ of peak. The state to be estimated (peak load) considers error-free values for measurements and pseudo-measurements derived from off-line power flows. Thus, "Case I - Peak" and "Case II - Peak" represent the expected maximum increments for the ISE. The flat start is used to initialize the estimator. As it can be seen from the figure, the voltages $e_{k}$ and $f_{k}$ from a previous load condition does not affect the maximum increment behavior. Therefore, the ISE is robust in that its convergence (for any tolerance) is not affected by voltages $e_{k}$ and $f_{k}$ obtained from a previously estimated state.

To further explore the robustness of the ISE, Fig. 8 presents the maximum increment in state variables at the convergence point $\left(\tau=10^{-5}\right)$ in 100 simulations for both "Case I $-90 \%$ " and "Case II - 90\%" considering additional random errors up to $\pm 30 \%$ for voltages $e_{k}$ and $f_{k}$. In all simulations, the ISE converged in 4 or less iterations. The quality of the corresponding estimated states can be verified in Fig. 9 and Fig. 10 where the maximum estimated errors in complex voltages and zero injections are negligible. Similar results were found when fixed variances were used, achieving convergence in no more than 5 iterations as well as quality estimations. Consequently, it can be concluded that the proposed ISE is robust enough to cope with a significant range of voltages $e_{k}$ and $f_{k}$ resulting from previous load levels.

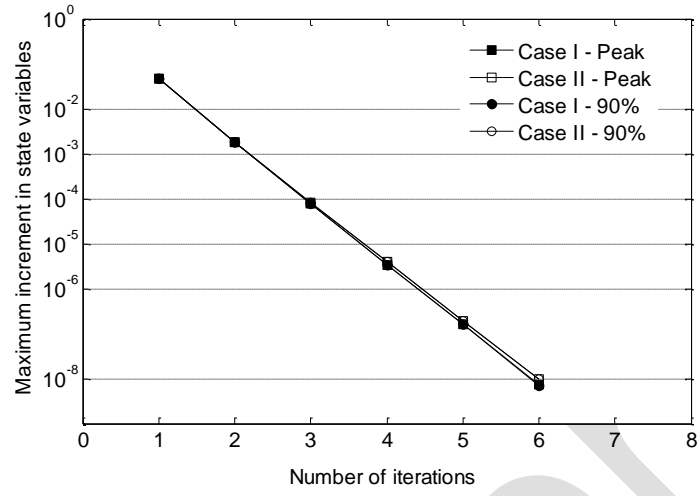

Fig. 7. Maximum increment in state variables per iterations for the ISE considering different values for voltages $e_{k}$ and $f_{k}$.

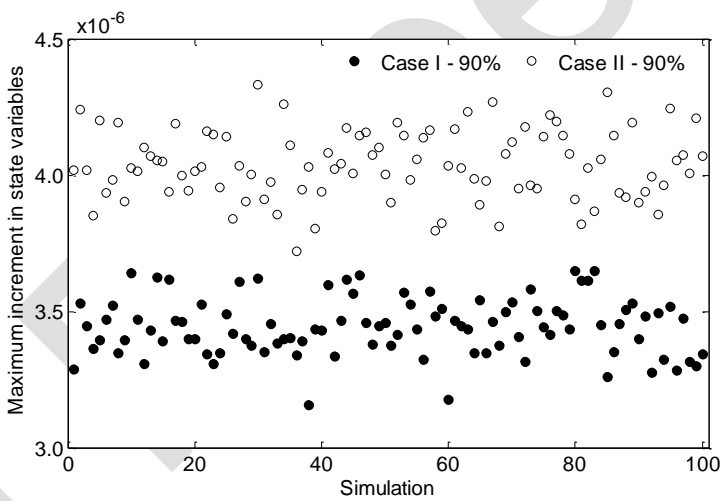

Fig. 8. Maximum increment in state variables in the convergence point per simulation. Random error up to $\pm 30 \%$ were included in $e_{k}$ and $f_{k}$.

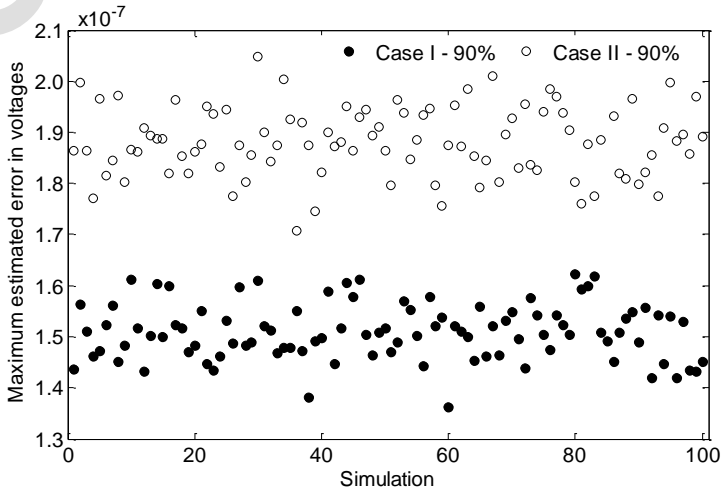

Fig. 9. Maximum estimated errors in voltages per simulation considering random errors up to $\pm 30 \%$ for voltages $e_{k}$ and $f_{k}$.

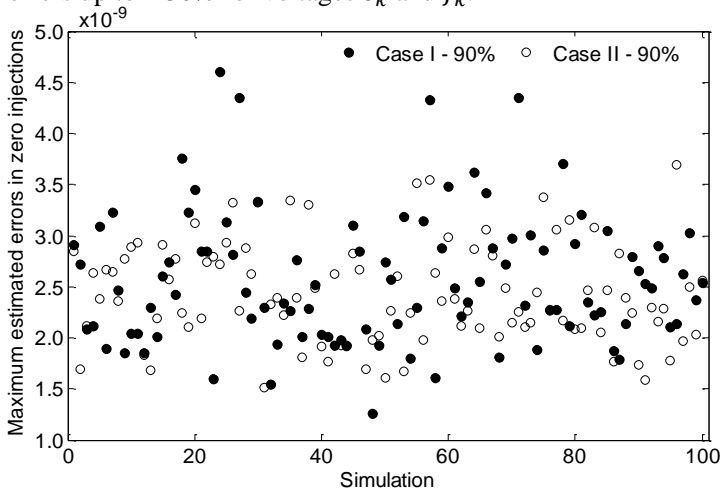

Fig. 10. Maximum estimated errors in zero injections per simulation considering random errors up to $\pm 30 \%$ for voltages $e_{k}$ and $f_{k}$. 


\section{F. Consistency Analysis}

In this section, the consistency of the ISE is assessed and compared with a similar SE that does not consider the covariances of the equivalent current measurements (referred to as ISE*). A Monte Carlo analysis is carried out considering 2,000 simulations for each SE and both measurements sets (Case I and Case II). Three confidence levels $(\alpha), 95,97.5$ and $99 \%$, are investigated. Voltages $e_{k}$ and $f_{k}$ are obtained for $90 \%$ of the peak load (a previous state) while measurements and pseudo-measurements correspond to the peak load (state to be estimated). Random errors following a normal distribution for voltage measurements, power flow measurements and injection pseudo-measurements are considered with values of up to $\pm 0.5, \pm 3$ and $\pm 30 \%$, respectively. A $\tau=10^{-5}$ is adopted.

Table II and Table III show the results of the consistency analysis for the proposed ISE and the ISE* considering the first (Case I) and second (Case II) sets of measurements, respectively. As it can be observed in both tables, the average consistency index, $\mu$, is close to 534 which corresponds to the total number of state variables (822) minus the number of zero injections $(48 \times 6=288)$, i.e., indicating the consistency of both SEs. However, if the covariances between real and imaginary parts of the equivalent current measurements are taken in account, as in the proposed ISE, the number of simulations where $\mu$ is out of bounds is reduced approximately by half. For instance, considering a confidence level $\alpha=95 \%$, this number reduces from 10.4 to $5.3 \%$ in Case I and from 10.2 to $5.3 \%$ in Case II. This is a clear indication that the consistency is significantly improved.

Additionally, even in the presence of random errors in measurements and pseudo-measurements, the ISE reaches convergence in up to 4 iterations. Furthermore, the average estimated errors in zero injections are negligible indicating that zero injections are satisfied. Finally, with an increased number of monitors, i.e., from Case I to Case II, the average voltage squared error, $\varphi=\left(\bar{E}_{n}-\hat{E}_{n}\right)^{T}\left(\bar{E}_{n}-\hat{E}_{n}\right)$, decreases sharply, demonstrating that, as expected from a consistent SE, the estimated state is improved.

Fig. 11 shows the voltage magnitudes for phase A of the test system estimated with the ISE for both measurements sets (Case I and Case II). The voltages for the 2,000 simulations of the Monte Carlo analysis are shown. Considering the random errors for measurements and pseudo-measurements, the voltages estimated for the peak load are expected to be within the range. As observed from Case I to Case II, the more monitors available the narrower the voltage range for each bus, thus resulting in more precise estimates.

\section{G. Computational Aspects}

Similar to traditional power flows, coefficient matrices need to be built and factorized to then use the resulting matrices in the solution process. These are the most time consuming tasks in state estimation algorithms [3], particularly for large three-phase distribution systems. This computational burden is, in general, related to the proportion of non-zero elements of these matrices. Therefore, this section
TABLE II

Consistency Analysis with Case I Measurements

\begin{tabular}{|c|c|c|}
\hline & ISE & ISE* \\
\hline $\begin{array}{c}\text { Simulations out of } \\
\text { bounds for } \alpha=95 \%\end{array}$ & $107 / 2000(5.3 \%)$ & $208 / 2000(10.4 \%)$ \\
\hline $\begin{array}{c}\text { Simulations out of } \\
\text { bounds for } \alpha=97.5 \%\end{array}$ & $50 / 2000(2.5 \%)$ & $111 / 2000(5.5 \%)$ \\
\hline $\begin{array}{c}\text { Simulations out of } \\
\text { bounds for } \alpha=99 \%\end{array}$ & $18 / 2000(0.9 \%)$ & $66 / 2000(3.3 \%)$ \\
\hline Average $\mu$ & 536.1 & 535.7 \\
\hline $\begin{array}{c}\text { Average number of } \\
\text { iterations }\end{array}$ & 4 & 4 \\
\hline $\begin{array}{l}\text { Average estimated error } \\
\text { in zero injections [pu] }\end{array}$ & $2.55 \times 10^{-9}$ & $2.53 \times 10^{-9}$ \\
\hline Average $\varphi[\mathrm{pu}]$ & $2.53 \times 10^{-4}$ & $2.58 \times 10^{-4}$ \\
\hline
\end{tabular}

\begin{tabular}{c|c|c}
\hline & ISE & ISE* \\
\hline $\begin{array}{c}\text { Simulations out of } \\
\text { bounds for } \alpha=95 \%\end{array}$ & $108 / 2000(5.4 \%)$ & $204 / 2000(10.2 \%)$ \\
\hline $\begin{array}{c}\text { Simulations out of } \\
\text { bounds for } \alpha=97.5 \%\end{array}$ & $59 / 2000(2.9 \%)$ & $116 / 2000(5.8 \%)$ \\
\hline $\begin{array}{c}\text { Simulations out of } \\
\text { bounds for } \alpha=99 \%\end{array}$ & $26 / 2000(1.3 \%)$ & $60 / 2000(3.0 \%)$ \\
\hline Average $\mu$ & 536.9 & 536.1 \\
\hline $\begin{array}{c}\text { Average number of } \\
\text { iterations }\end{array}$ & 4 & 4 \\
\hline $\begin{array}{c}\text { Average estimated error } \\
\text { in zero injections [pu] }\end{array}$ & $2.55 \times 10^{-9}$ & $2.54 \times 10^{-9}$ \\
\hline Average $\varphi$ [pu] & $0.39 \times 10^{-4}$ & $0.41 \times 10^{-4}$ \\
\hline
\end{tabular}

* Covariances of the equivalent measurements are not considered.

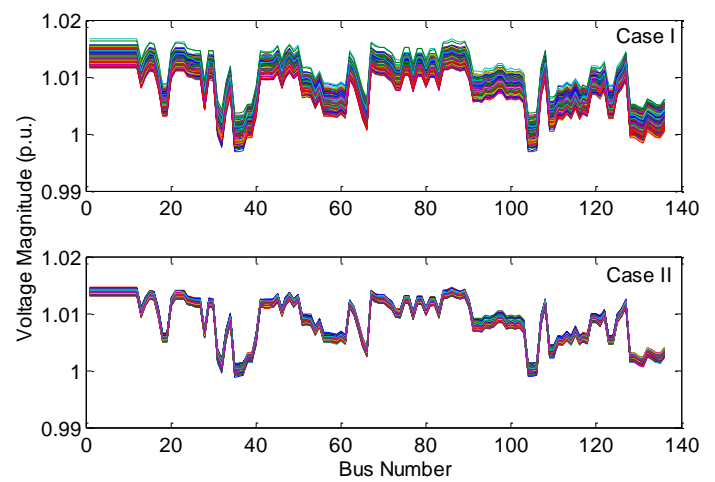

Fig. 11. Voltages magnitudes for phase A estimated with the ISE for the 2,000 simulations of the Monte Carlo analysis.

compares the computational performance of the proposed ISE with that of the AMB NE and AMB NE/C by evaluating the non-zero elements of the corresponding matrices.

Table IV presents the number of non-zero elements of the coefficient matrices as well as the lower and upper triangular matrices (LU factorization from Matlab [22]) for the three SEs and for both measurements sets (Case I and Case II). For the proposed ISE, the two coefficient matrices, $H_{o o}$ and $A^{T} W A$, are shown separately. The coefficients matrices for the AMB $\mathrm{NE}$ and the AMB NE/C are shown in the Appendix.

Despite the larger total number of non-zero elements in the two coefficient matrices of the ISE, which can demand more computational effort for their factorization, the number of nonzero elements in the corresponding lower and upper triangular 
TABLE IV

Size and number of non-zero elements in coefficient matrices and Lower and Upper triangular Matrices.

\begin{tabular}{|c|c|c|c|c|c|}
\hline \multirow{2}{*}{\multicolumn{2}{|c|}{ Matrices }} & \multirow{2}{*}{ Size } & \multicolumn{3}{|c|}{ Number of Non-Zero Elements } \\
\hline & & & Coefficient & Lower & Upper \\
\hline \multicolumn{6}{|c|}{ Case I } \\
\hline \multirow{2}{*}{ ISE } & $A^{T} W A$ & $536 \times 536$ & 38,704 & 103,101 & 106,644 \\
\hline & $H_{o o}$ & $288 \times 288$ & 2,802 & 3,493 & 3,421 \\
\hline \multicolumn{2}{|c|}{$A M B N E$} & $824 \times 824$ & 26,710 & 159,948 & 162,533 \\
\hline \multicolumn{2}{|c|}{$A M B N E / C$} & $1116 \times 1116$ & 27,247 & 298,076 & 290,894 \\
\hline \multicolumn{6}{|c|}{ Case II } \\
\hline \multirow{2}{*}{ ISE } & $A^{T} W A$ & $536 \times 536$ & 38,704 & 102,840 & 107,187 \\
\hline & $H_{o o}$ & $288 \times 288$ & 2,802 & 3,493 & 3,421 \\
\hline \multicolumn{2}{|c|}{$A M B N E$} & $824 \times 824$ & 26,798 & 159,950 & 162,650 \\
\hline \multicolumn{2}{|c|}{$A M B N E / C$} & $1116 \times 1116$ & 27,665 & 289,358 & 287,935 \\
\hline
\end{tabular}

matrices are much smaller than in the other AMB SEs, i.e., resulting in more computationally efficient solution process at each iteration. For instance, in Case I the total number of nonzero elements in the lower triangular matrices of the ISE is two thirds and one third of those from the AMB NE and the $\mathrm{AMB} \mathrm{NE} / \mathrm{C}$, respectively. The same can be observed for the upper triangular matrices and both measurements sets. Consequently, in general, the ISE will be at least as computationally competitive as the AMB NE and AMB NE/C. Additionally, since the coefficient matrices are built and factorized just once for the ISE, it is computationally much more efficient than those SEs whose coefficient matrices are built and factorized every iteration, such as the traditional SE.

\section{DISCUSSION}

To determine the most suitable operation of network elements and participants, future distribution systems are likely to use advanced Distribution Management Systems (DMS) which in turn require trustworthy visibility across the voltage levels [1], [2]. The computational features of the proposed ISE makes it a competitive alternative to carry out large-scale three-phase estimates not only to increase visibility but also to improve the reliability of information extracted from measurements that will come from current and future network elements and participants (e.g., smart meters, smallto-medium scale distributed energy resources, etc.). More accurate and reliable estimates within advanced DMS will enable a myriad of Smart Grid-related applications.

In this context, some key features and implementation aspects of the proposed ISE are discussed below.

\section{A. Robustness of the ISE}

Although the WLS is not a robust approach it is the most widely used to estimate the state of power systems. This is because, in general, WLS SEs present good convergence features for almost all system topologies and levels of monitoring. However, this approach can experience convergence problems in very particular cases. The main sources for convergence problems include [3], [4], [8]:

- large number of injections measurements;

- the presence of branches with low and large impedances connected to the same bus;

- the very contrasting weights associated to pseudomeasurements and virtual measurements (as zero injections).
In this paper, the zero injections are represented without requiring the high weights usually adopted in WLS SEs, thus contributing to improve the robustness of the ISE. Additionally, it was proposed an original approach to represent magnitude voltage measurements, which helps to improve the convergence features and, therefore, the robustness of the ISE.

Nonetheless, despite the fact that the proposed ISE is aimed at tackling some important shortcomings of AMB SEs, it cannot be classified as a robust approach [3], [4].

\section{B. Modelling of Network Elements and Loads}

Since the proposed ISE handles network elements in the same way as the traditional SE (or power flow), the corresponding models can be directly adopted. Although the involved variables change from the traditional SE to the AMB SEs (e.g., power into currents, etc.), the models and equations -even for especial devices- remain the same.

Loads are typically treated by SEs as part of the "external" network (i.e., represented by measurements) and, thus, the corresponding connections do not need to be modelled to estimate the state [3], [4]

\section{System Topology}

In classical state estimation, a network topology processor provides the system topology based on the status of switching devices and a complete description of the network elements [3]. This topology is assumed correct. However, if the topology contains errors, the estimated state can be seriously compromised [3], [4]. In order to handle this, the proposed ISE can be adapted to model breakers/switches and find, simultaneously, the best estimate for the system topology and state variables [3], [15], [16], [23], [24]. The main concern in modelling switching devices is that the number of state variables as well as the required number of measurements can increase significantly, enlarging the computational effort and the costs associated to the measurement system [3].

The proposed ISE is also able to cater for different types of topology, even for completely meshed systems. Although the sparsity of the coefficient matrices will reduce as the number of meshes increase, these matrices will still be built and factorized just once. Therefore, the proposed ISE will continue to be a very attractive alternative from the computational point of view even for meshed systems.

\section{Lack of Measurements}

In cases where the only measurements available are threephase power values (e.g., distributed generation), the corresponding phase values will be considered as pseudomeasurements with magnitudes equal to a third of the total power (i.e., three-phase value is split equally among phases). In addition, variances must be adequately specified per phase (as described in section III.G).

The real-time implementation of SEs is likely to face challenges from the malfunction of monitoring devices and/or the communication infrastructure [3]. Nonetheless, the resulting loss of measurement data from one instant to another can be handled by, for instance, using values calculated from the last estimates or adopting the last measured values as pseudo-measurements. 


\section{CONCLUSIONS}

This paper presented an improved AMB SE (ISE) for threephase medium voltage distribution systems that enhances robustness and consistency. The state variables are the real and imaginary parts of the bus voltages, while the actual measurements are converted into equivalent currents and voltages. The zero injections are included through a linear non-weighted procedure preventing convergence problems. The resulting coefficient matrices are constant and positive definite requiring only simple and classical factorization techniques. A new approach based on phasor rotation is also proposed to obtain the equivalent voltage measurements improving significantly the convergence of the ISE. Furthermore, it is proposed the usage of the covariances between the real and imaginary parts of the equivalent current measurements in order to improve the consistence of the ISE.

The case studies, based on a real UK medium voltage system, have considered perfect measurements as well as measurements containing random errors. Through a Monte Carlo analysis, the proposed ISE have shown to be robust and statistically consistent. Indeed, the ISE presents very good convergence features and provides estimated values and variances in agreement with the specified measurements and variances. Additionally, comparisons with the AMB NE and the AMB NE/C show that the proposed three-phase ISE is significantly more robust and consistent as well as being computationally very competitive for smart distribution systems applications.

Finally, it is important to highlight that the proposed improvements can be easily applied to any AMB SE formulation. For instance, the convergence and consistency of the AMB NE/C SE can be improved by adopting the proposed approach to obtain the equivalent voltage measurements and the covariances of the equivalent current measurements.

\section{ACKNOWLEDGEMENT}

The authors would like to thank Electricity North West Limited (ENWL), UK, for providing the MV system data.

\section{APPENDIX}

The coefficient matrices for the AMB NE and AMB NE/C SEs considered in Section V-E are (22) and (23), respectively. $W_{o}$ is a diagonal matrix containing the weights for the real and imaginary parts of the zero injections.

$$
\begin{gathered}
{\left[\begin{array}{ll}
H_{n}^{T} & H_{o}^{T}
\end{array}\right]\left[\begin{array}{cc}
W & 0 \\
0 & W_{o}
\end{array}\right]\left[\begin{array}{l}
H_{n} \\
H_{o}
\end{array}\right]} \\
{\left[\begin{array}{cc}
H_{n}^{T} W H_{n} & H_{o}^{T} \\
H_{o} & 0
\end{array}\right]}
\end{gathered}
$$

\section{REFERENCES}

[1] J. Wu, Y. He and N. Jenkins, "A robust state estimator for medium voltage distribution networks", Power Systems, IEEE Transactions on, vol.28, no.2, pp.1008-1016, May 2013.

[2] Y. Xiang, P. F. Ribeiro and J. F. G. Cobben, "Optimization of StateEstimator-Based Operation Framework Including Measurement Placement for Medium Voltage Distribution Grid", Smart Grid, IEEE Transactions on, vol.5, no.6, pp.2929-2937, Nov. 2014.

[3] A. Monticelli, State Estimation in Electric Power Systems: A Generalized Approach. Kluwer, 1999.

[4] A. Abur and A. Gómez-Expósito, Power System State Estimation: Theory and Implementation. Marcel Dekker, 2004.

[5] D. A. Haughton and G. T. Heydt, "A Linear State Estimation Formulation for Smart Distribution Systems", Power Systems, IEEE Transactions on, vol.28, no.2, pp.1187-1195, May 2013.

[6] M. C. de Almeida, E. N. Asada, E.N and A. V. Garcia, "Effects of load imbalance and system asymmetry on three-phase state estimation", PES General Meeting, 2006 IEEE, 18-22 June 2006.

[7] J. C. Das, Power System Analysis: Short-Circuit Load Flow and Harmonics. CRC Press, 2011.

[8] W. M. Lin, and J. H. Teng, "State estimation for distribution systems with zero-injection constraints", Power Systems, IEEE Transactions on, vol. 11. no.1, pp.518-524, Feb. 1996.

[9] Y. Guo, W. Wenchuan, B. Zhang and H. Sun, "An Efficient State Estimation Algorithm Considering Zero Injection Constraints", Power Systems, IEEE Transactions on, vol.28, no.3, pp.2651-2659, Aug. 2013.

[10] L. Holten, A. Gjelsvik, S. Aam, F. F Wu and W.-H.E. Liu, "Comparison of different methods for state estimation", Power Systems, IEEE Transactions on, vol.3, no.4, pp.1798-1806, Nov 1988.

[11] P. M. De Oliveira-De Jesus and A. A. Rojas Quintana, "Distribution System State Estimation Model Using a Reduced Quasi-Symmetric Impedance Matrix," IEEE Transactions on Power Systems, vol. 30, no. 6, pp. 2856-2866, Nov. 2015.

[12] G. N. Korres and T. A. Alexopoulos, "A Constrained Ordering for Solving the Equality Constrained State Estimation", Power Systems, IEEE Transactions on, vol.27, no.4, pp.1998-2005, Nov. 2012.

[13] C. N. Lu, J. H. Teng, and W. H. E. Liu, "Distribution system state estimation", Power Systems, IEEE Transactions on, vol.10, no.1, pp.229-240, Feb. 1995.

[14] W-M. Lin and J. H. Teng, "Distribution fast decoupled state estimation by measurement pairing", Generation, Transmission and Distribution, IEE Proceedings of, vol.143. no.1, pp.43-48, Jan. 1996.

[15] H. B. Sun, F. Gao, K. Strunz, B. M. Zhang, and Q. X. Li, "Analog digital power system state estimation based on information theory-Part II: Implementation and application," IEEE Trans. Smart Grid, vol. 4, no. 3, Sep. 2013.

[16] H. B. Sun, F. Gao, K. Strunz, and B. M. Zhang, "Analog digital power system state estimation based on information theory-Part I: Theory," IEEE Trans. Smart Grid, vol. 4, no. 3, Sep. 2013.

[17] M. C. de Almeida, R. Schincariol and F. L. Ochoa, "Assessing the Statistical Consistency of the AMB State Estimator in Distribution Systems", PES ISGT LATAM, 2015 IEEE, pp.585-590, Oct. 2015.

[18] R. Singh, B. C. Pal and R. A. Jabr, "Choice of estimator for distribution system state estimation", Generation, Transmission \& Distribution, IET, vol.3, no.7, pp.666-678, July 2009.

[19] M. Yucra, F. Schmidt and M. C. de Almeida, "Analysis of Bad Data Detection in Power System State Estimators Considering PMUs" PES General Meeting, 2015 IEEE, pp. 26-30 July 2015.

[20] C. Muscas, M. Pau, P. A. Pegoraro, and S. Sulis, "Effects of Measurements and Pseudomeasurements Correlation in Distribution System State Estimation", Instrumentation and Measurement, IEEE Transactions on, vol.63, no.12, pp.2813-2823, Dec. 2014.

[21] T. W. Anderson, An introduction to multivariate statistical analysis, New York: Willey, 1958.

[22] MATLAB Release R2012a, The MathWorks, Inc., Natick, Massachusetts, United States.

[23] K. A. Clements and A. S. Costa, "Topology error identification using normalized Lagrange multipliers," IEEE Transactions on Power Systems, vol. 13, no. 2, pp. 347-353, May 1998.

[24] G. N. Korres and P. J. Katsikas, "Identification of circuit breaker statuses in WLS state estimator," IEEE Transactions on Power Systems, vol. 17, no. 3, pp. 818-825, Aug 2002. 